\title{
Duplication of the Etrahepatic Bile Duct with a Benign Stricture
}

\author{
Masanori Matsuda ${ }^{1}$, Masataka Kikuyama ${ }^{1}$ and Tatsuki Ueda ${ }^{2}$
}

Key words: sclerosing cholangitis, steroid, IDUS, ERCP

(Intern Med 52: 2679-2680, 2013)

(DOI: 10.2169/internalmedicine.52.1297)

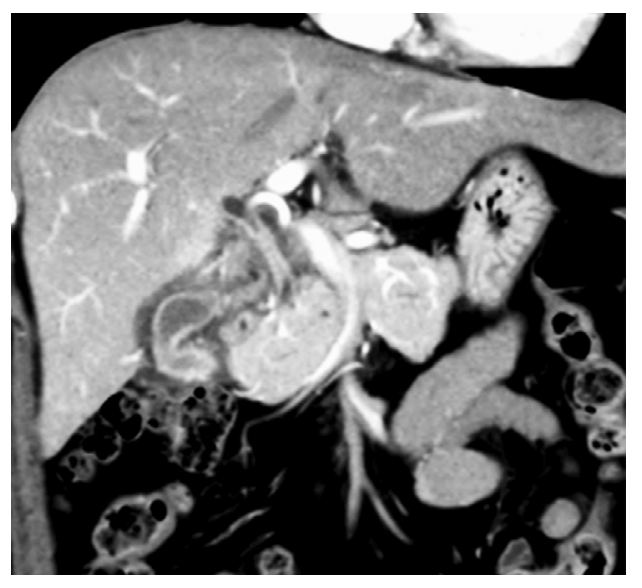

Picture 1.

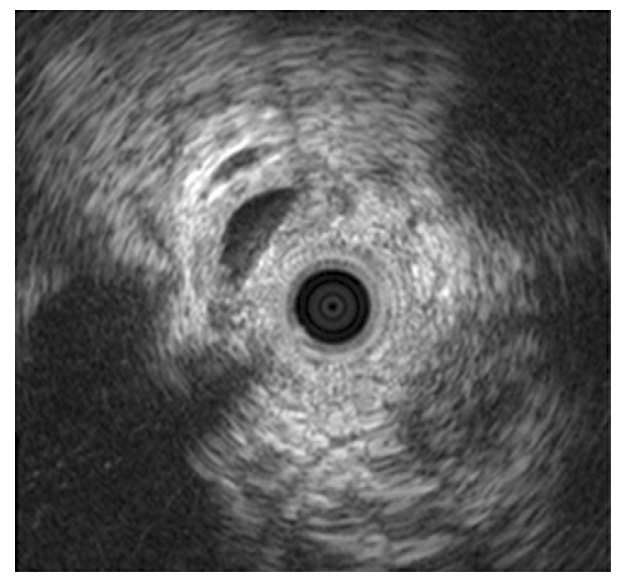

Picture 3.

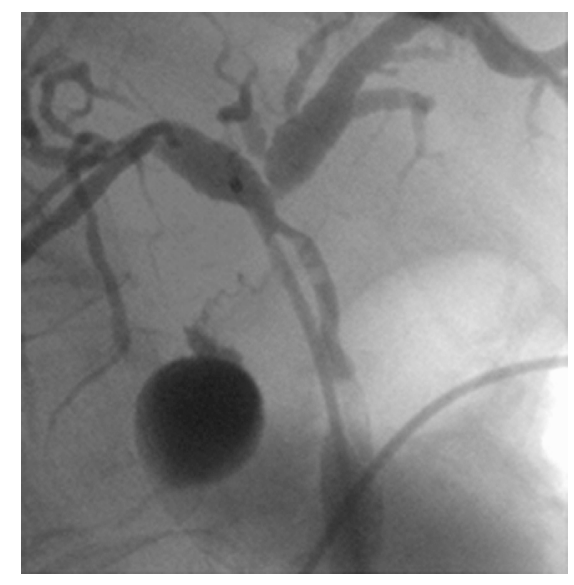

Picture 2.

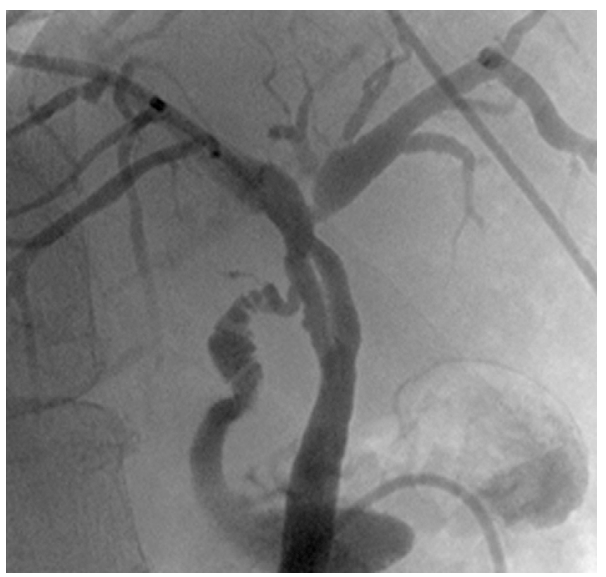

Picture 4.
A 50-year-old woman presented with epigastralgia. A blood examination showed liver dysfunction. The IgG4 level was within the normal limits. CT (Picture 1) revealed a thickened wall of the extrahepatic bile duct. ERCP (Picture 2) showed paired extrahepatic bile ducts with a stric- ture. Intraductal ultrasonography (IDUS, Picture 3) demonstrated a regularly thickened wall of each duct with a layer structure. A biopsy and cytology of the strictured portion did not show malignancy. A nasobiliary tube was placed in the right intrahepatic bile duct through one of the paired bile

${ }^{1}$ Department of Gastroenterology, Shizuoka General Hospital, Japan and ${ }^{2}$ Department of Gastroenterology, Kyoto University Hospital, Japan Received for publication July 4, 2013; Accepted for publication July 5, 2013

Correspondence to Dr. Masataka Kikuyama, Kikuyama110@yahoo.co.jp 
ducts. Oral steroids were administered for two weeks, and the stricture of the extrahepatic bile duct resolved (Picture 4).

Our case is rare and was classified as a type V duplication of the extrahepatic bile duct (1). If the serum IgG4 level is elevated, making the diagnosis of bile duct stricture as benign is not difficult. In patients with a normal IgG4 level, steroid administration can contribute to the diagnosis.
The authors state that they have no Conflict of Interest (COI).

\section{Reference}

1. Choi E, Byun JH, Park BJ, Lee MG. Duplication of the extrahepatic bile duct with anomalous union of the pancreaticobiliary ductal system revealed by MR cholangiopancreatography. BJR 80: e150-e154, 2007.

(C) 2013 The Japanese Society of Internal Medicine http://www.naika.or.jp/imonline/index.html 